\title{
La alineación natural de la rodilla y su corrección protéica
}

PICHEL MOURE, C.M.

Servicio de Traumatología y Cirugía Ortopédica.

Hospital do Meixoeiro Vigo

\section{RESUMEN}

La reconstrucción artroplástica pretende reproducir la alineación normal de la articulación de la rodilla. Sin embargo la normalidad se encuentra pobremente definida al existir importantes variaciones interindividuales en los limitados estudios disponibles. En este artículo se examinan las posibles causas y consecuencias quirúrgicas de ésa variabilidad desde una perspectiva geométrica y biomecánica. Con este objetivo se midieron los ángulos que definen la alineación longitudinal y transversal de la rodilla y las dimensiones del esqueleto de los miembros inferiores sobre telerradiografías en bipedestación de 30 voluntarios asintomáticos de ambos sexos. Los datos fueron procesados estadísticamente, analizando la influencia del sexo y de la diversidad constitucional de las dimensiones de la cintura pélvica y de los miembros inferiores sobre las variaciones de la alineación trasversal y longitudinal de la rodilla. Se encontró una gran variabilidad en la mayoría de los parámetros medidos -el ángulo femorotibial varió entre $6^{\circ}$ de valgo y $7^{\circ}$ de varo- y diferencias muy significativas entre el grupo masculino y femenino en dicho ángulo y en la inclinación de la superficie articular tibial que parecieron guardar relación con las proporciones entre la longitud de los miembros inferiores y la anchura de la pelvis. Sin embargo, las condiciones de carga de las distintas rodillas permanecieron relativamente uniformes. Estos resultados inducen a pensar que la variabilidad natural de la alineación del miembro inferior puede obedecer al objetivo de mantener sobre la rodilla solicitaciones mecánicas adaptadas a las características antropométricas individuales.

Por ésta razón se sugieren respetar la alineación natural durante la reconstrucción artroplástica y, orientación individualizada de los componentes protésicos.

Palabras clave: Alineación de la rodilla, artroplastia de la rodilla, alineación protésica, biomecánica, antropometría, prótesis.

\section{SUMMARY}

The artroplastic reconstruction pretends to reproduce the normal alineation of the knee joint. Nevertheless the normality is poorly defined because of the existance of interindividual variations in the limitted studies seen. In this article the posible causes and surgical consequences of this variability are examined, from a geomethric and biomechanical perspective. With this object the angles that define the alineation of the knee were measured and the dimension of the inferior skeleton over teleradiographies of 30 bipedestian volunteers of both sexes. The results were statistically processed, analyzing the influnce of sex, constitutional diversity of the pelvic waist and of the lower members over the variations of the transversal alineation and knee length. It was found that a great variability of the parametres measured - the femural-shin-bone angle varied between $6^{\circ}$ and $7^{\circ}$ - and significant differences between males and females of the mentioned angle and the shin-bone articular surface inclination seemed to keep close relation with the proportions of the lower members length and the pelvical width. However, the charging conditions of the different kness stayed relatively uniform. This results induce to think that the natural variability of the lower member alineation can obey to the object of maintaining on the knee mechanical solicitations addapted to the individual antropometrical characteristics. For this reason it is suggested to respect the natural alineation during the artroplastic reconstruction and, based in the regresion anlysis of the facts studied, we elaborated a guiding table for the individual orientation of the prosthetic components.

Key words: Knee alineation, knee artroplasty, prosthetic alineation, biomechanic, antropomethry, prosthesis.

\section{INTRODUCCIÓN}

Se supone que en condiciones normales el eje mecánico femoral y el eje mecánico tibial se prolongan en el plano frontal ${ }^{12,17}$, formando un ángulo de $0^{\circ}$, y que la interlínea articular se orienta perpendicularmente a ellos. En consecuencia, se subraya la necesidad de reproducir con precisión la misma disposición cuando se implantan prótesis de rodilla. 1,6,9,16,19,20,22 Se pretende así restablecer la alineación fisiológica de los miembros inferiores.

Tales afirmaciones pocas veces han sido comprobadas experimentalmente. Aunque los valores medios del ángulo femorotibial en los grupos de población normal estudiados independientemente pro Moreland ${ }^{21}$ en 1987 y $\mathrm{Hsu}^{7}$ en 1990 no difirieron significativamente de $0^{\circ}$, ambos trabajos subrayan que el ángulo femorotibial no es constante, sino que se comporta como una variable con una considerable dispersión en su distribución de frecuencias. El origen de esta variabilidad y su importancia potencial no fue analizada por dichos autores; sin embargo, sus observaciones presuponen que la recons- 
trucció artroplástica de la rodilla pretende poner en línea recta numerosos miembros inferiores que constitucionalmente poseerían un ángulo femorotibial distinto de $0^{\circ}$. Por otra parte, aunque una alineación manifiestamente anormal conduce al fracaso de la artroplastia $9,11,20$, no se ha podido afirmar una relación estrecha entre la alineación en $0^{\circ}$ y los resultados de mejor calidad ${ }^{11,24}$ por lo que basándose en la experiencia clínica muchos autores aconsejan una moderada sobrecorreción en valgo para las sustituciones tricompartimentales y una desviación del eje mecánico del miembro hacia el compartimento sustituído en las monocompartimentales ${ }^{13}$.

Existen también desacuerdos en cuanto a la orientación de los ejes transversales de la rodilla. Así frente a la concepción clásica de su perpendicularidad con los ejes longitudinales, estudios realizados en varones jóvenes encontraron un ángulo medial de $87^{\circ}$ entre la interlí-

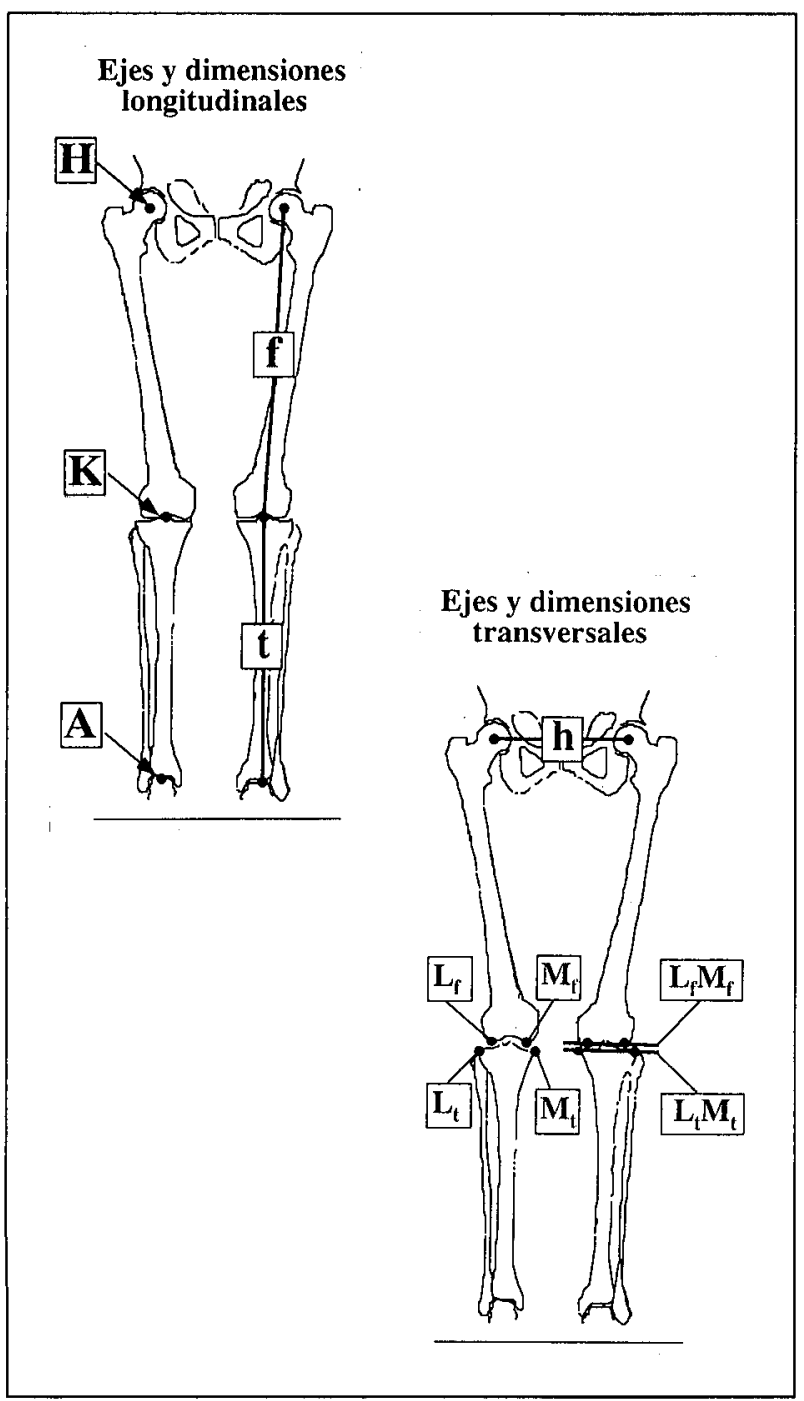

Figura 1.a) Se trazaron los ejes mecánicos femoral (f) y tibial (t) entre los centros mecánicos de la cadera $(H)$ y de la rodilla $(K)$ y entre éste último y el centro del tobillo (A) respectivamente y se midieron sus longitudes. 1. b) Se midió la distancia (h) o diámetro bifemoral y se dibujaron la línea bicondílea $\left(\mathrm{L}_{\mathrm{f}} \mathrm{M}_{\mathrm{f}}\right)$ y la línea bituberositaria (Ltmt). nea articular y el eje tibial ${ }^{8,21}$; lo que dió pie a la propuesta de situar los componenetes protésicos tibiales con una inclinación medial de $3^{\circ}$, disposición a la que sus autores denominaron «alineación anatómica»15.

Estos antecedentes ponen en evidencia la existencia de discrepancias ente las predicciones conceptuales y los resultados prácticos que justificaron el presente estudio; cuyo objetivo principal consistió en determinar la alineación de los ejes longitudinales y transversales del miembro inferior en sujetos asintomáticos prestando especial atención a los posibles orígenes de la variabilidad de sus valores. Para ello se investigó la relación de las variaciones encontradas con el lado, el sexo, las dimensiones transversales de la pelvis y la longitud de los huesos principales del miembro inferior (fémur y tibia). Finalmente se estudiaron algunas de sus cosecuencias mecánicas y se obtuvieron valores de la alineación ajustados a proporciones antropométricas individuales como guía para la reconstrucción quirúrgica.

\section{MATERIAL Y MÉTODOS}

Se estudiaron 30 voluntarios asintomáticos, 16 varones y 14 mujeres, con una edad mínima de 13 y una máxima de 49 años, en actitud de bipedestación equilibrada; practicándoseles telerradiografías de los miembros inferiores mientras mantenían entre éstos la máxima aproximación posible sin que se produjese contacto perceptible entre las rodillas o los tobillos. En cada telerradiografía se identificaron los centros mecánicos de las cadera $(\mathrm{H})$, rodillas $(\mathrm{K})$ y tobillos $(\mathrm{A})$; se trazaron los ejes mecánicos femorales (f) y tibiales ( $t$ ), la linea horizontal que une ambos centros de las caderas o diámetro bifemorales (h) y se midieron sus longitudes. Se dibujaron también dos líneas tangentes a los cóndilos femorales (línea bicondílea, LMf) y platillos tibiales (línea bituberositaria, LMt) respectivamente (fig 1). La alineación longitudinal del miembro inferior fué estimada midiendo el ángulo existente entre el eje femoral y la prolongación proximal del eje tibial, al que llamamos ángulo femorotibial $(\alpha)$. Cuando el eje tibial sobrepasó por fuera al eje femoral (varo) se le atribuyó un valor positivo y negativo en caso contrario (valgo). La orientación transversal de la interlínea articular se estimó midiendo el ángulo formado lateralmente por el eje mecánico femoral con la tangente distal a los condilos femorales y el delimitado medialmente por el eje mecánico tibial y la linea bituberositaria. Dichos ángulos se denominaron respectivamente ángulo condíleo $(\varphi)$ y ángulo tibial $(\tau)$. Para medir el paralelismo de la interlínea articular radiológica se obtuvo, a partir de relaciones geometricas, el valor del ángulo formado entre las líneas bituberostaria y bicondílea, al que se designó ángulo de convergencia articular $(\beta)$. Su valor resultó positivo o negativo según ambas lineas convergieron en dirección medial o lateral respectivamente (fig 2). Las longitudes se expresaron en milimetros y los ángulos en grados sexagesimales. Se analizaron también como variables las relaciones con el diámetro bifemoral $(h)$ de la longitud del fémur $(\mathrm{f} / \mathrm{h})$, de la longitud de la tibia $(\mathrm{t} / \mathrm{h})$ $y$ de la suma de ambas $((f+t) / h)$. Las dimensiones $y$ ángulos medidos fueron sometidos a análisis estadístico comparando los valores correspondientes a rodillas derechas e izquierdas y las correspondientes a mujeres y 


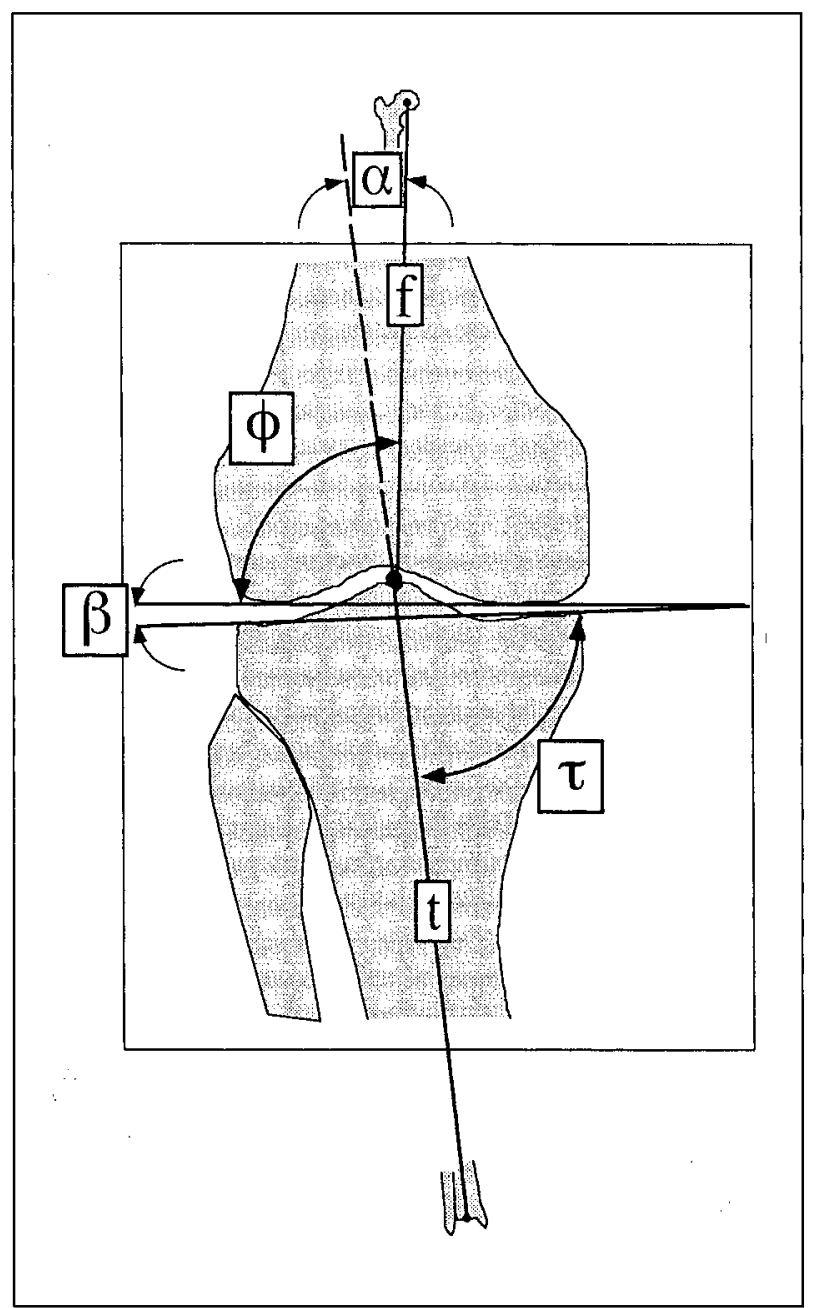

Figura. 2. El ángulo femorotibial $(\alpha)$ se consideró positivo cuando la prolongación del eje tibial pasó por fuera del eje femoral (varo) y negativo en caso contrario (valgo). El ángulo condíleo $(\varphi)$ se identificó con el formado lateralmente por el eje femoral y la línea bicondílea y el ángulo tibial $(\tau)$ con el formado medialmente entre el eje tibial y la línea bituberositaria. Se designó «ángulo de convergencia articular» $(\beta)$ al comprendido entre la línea bicondílea y la línea bituberositaria, cuyo valor $\beta=\tau+\alpha-\varphi$, deducido de las relaciones geométricas de la figura, fue positivo cuando la interlínea convergió medialmente y negativo en caso contrario.

varones mediante el test de la $t$ de Student para comparación de muestras. A fin de profundizar en las causas de la dispersión de valores y de las diferencias sexuales encontradas, las variables correspondientes a las muestras de mujeres, varones y ambos conjuntamente se sometieron a un análisis de regresión lineal.

En las 22 radiografías mas recientes de la serie (26 varones y 18 mujeres; 44 rodillas) se midió el ángulo formado lateralmente por cada eje tibial ( $\mathrm{t}$ ) con la horizontal ó ángulo de inclinación tibial $(\varphi)$, se calculóla distancia (z) de la línea de acción de la reacción de apoyo en el pie al centro de la rodilla y se estimó el valor que adquiría esta variable $\left(\mathrm{z}_{0}\right)$ suponiendo que se corrigiese la alineación a $0^{\circ}$ y se mantuviese la misma posición de las caderas. Asumiendo que en las condiciones de equilibrio estático en que se realiza la radiografía la reacción de apoyo en cada pie es vertical y pasa por el

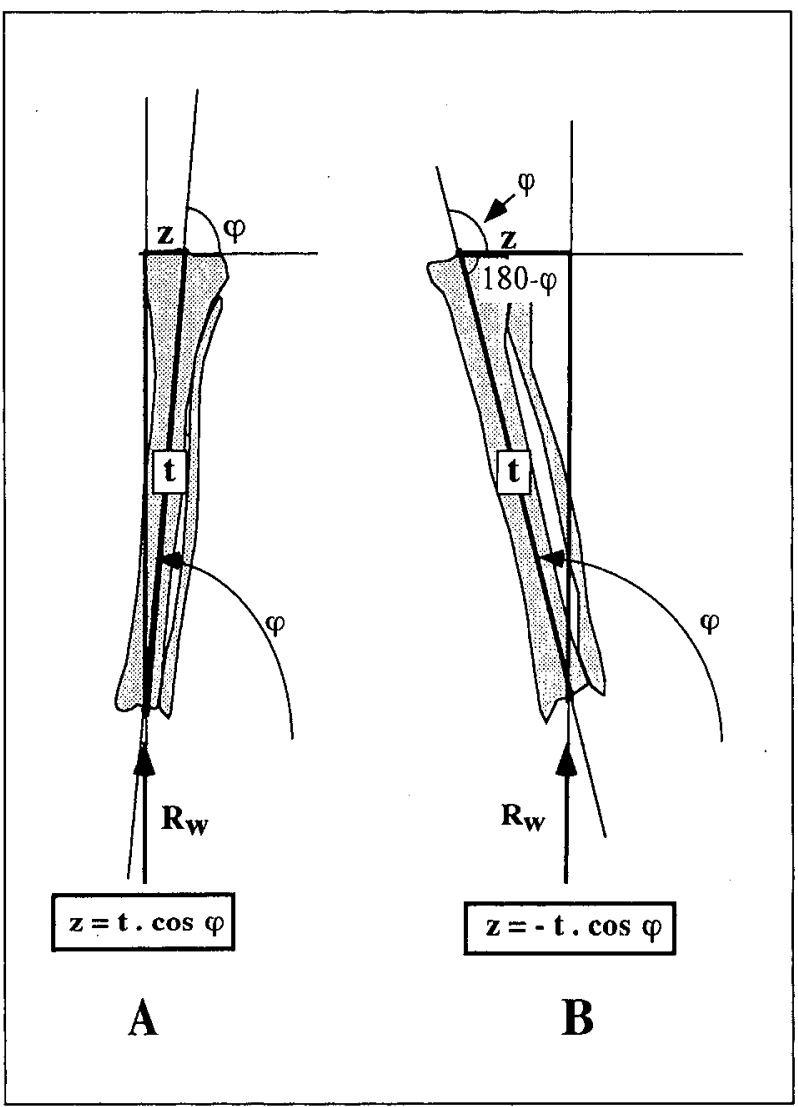

Figura 3. Permaneciendo de pie en equilibrio, la línea de acción de la reacción de apoyo $(\mathbf{R w})$ es vertical por lo que, asumiendo que pasa por el centro del tobillo, su distancia al centro de la rodilla (z) es función de la longitud de la tibia $(t)$ y de su inclinación respecto al suelo $(q)$; inclinación que depende a su vez de la dirección del fémur y del ángulo femorotibial. Se asignaron a $z$ valores positivos o negativos según se situase medial o lateral al centro de la rodilla respectivamente.

centro del tobillo ${ }^{14}$ se dedujeron las distancias $\mathrm{z}$ y $\mathrm{z}_{0}$ de acuerdo a las relaciones $\mathrm{z}=\mathrm{t} . \cos \varphi$, (fig. 3) $\mathrm{y} \mathrm{z}_{0}=\mathrm{t}$. $\cos (\varphi+\alpha)$, (fig. 4). Se calculó la media de los valores $(\mathrm{f}+\mathrm{t}) / \mathrm{h}$, se agruparon separadamente los que no alcanzaron su valor $\left(m_{1}\right)$ y los que lo superaron $\left(m_{2}\right)$ y se compararon entre ambas muestras (t-test, doble cola) los valores medios de los ángulos; de las dimensiones lineales y de las distancias de la reacción de apoyo en condiciones naturales y tras la alineación simulada a $0^{\circ}$. Esta parte del estudio se completó con un análisis de regresión de las variables $\mathrm{z}$ y $\mathrm{z}_{0}$ con la relación $(\mathrm{f}+\mathrm{t}) / \mathrm{h}$.

Introduciendo variaciones crecientes en la proporcion $(\mathrm{f}+\mathrm{t}) / \mathrm{h}$ se obtuvieron a partir de las funciones de regresión los valores ajustados correspondientes de los ángulos femorotibial, condíleo, tibial y de convergencia articular. Finalmente, utilizando las relaciones geométricas que mantienen entre sí ( $\varphi-\alpha=\tau-\beta$; ver fig. 3$)$, se calcularon los valores de éstos ángulos en el supuesto de paralelismo de la interlínea $\left(\beta=0^{\circ}\right)$ y se redondearon los resultados.

\section{RESULTADOS}

La edad media de los voluntarios estudiados fué de 


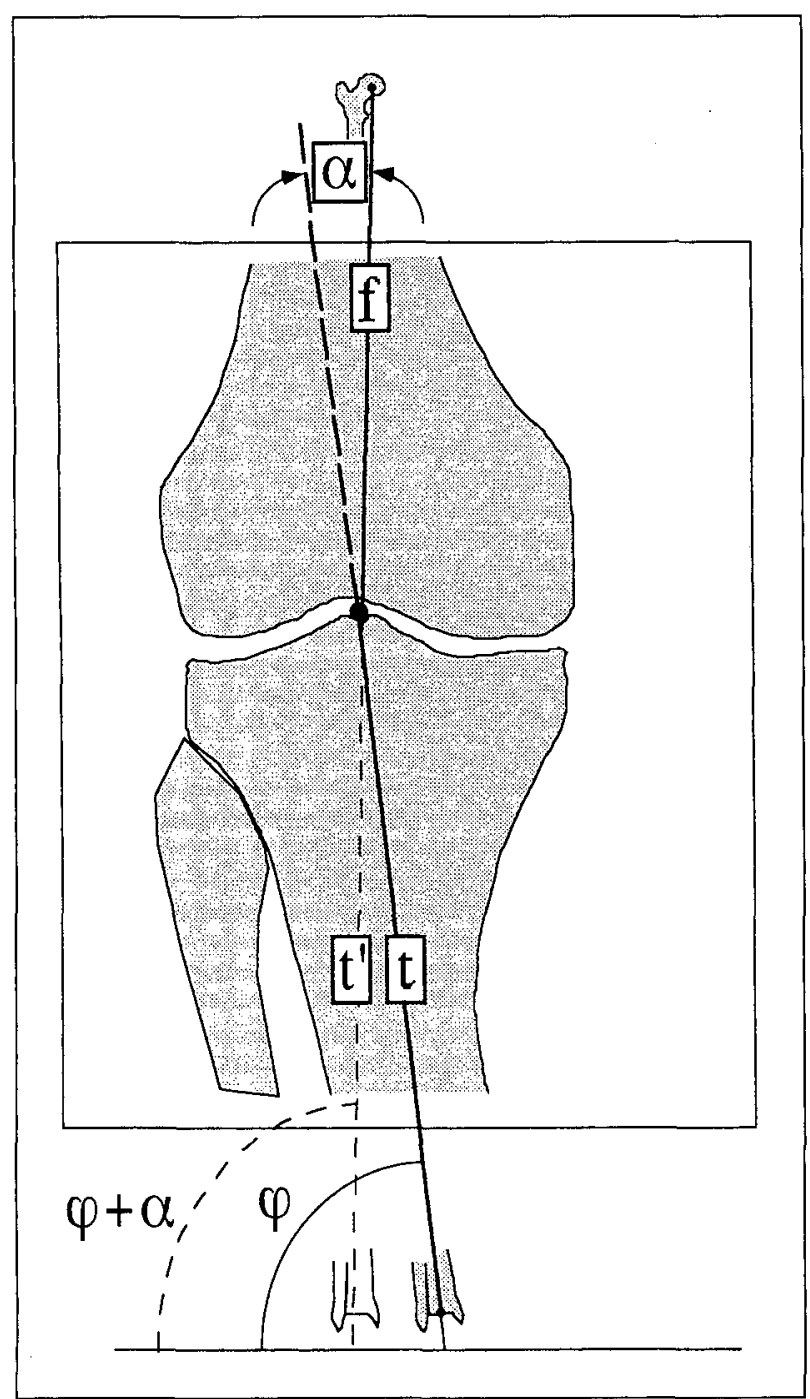

Figura 4. Si se corrige la alineación entre el eje mecánico femoral y tibial de modo que ambos se encuentren en prolongación, para la misma orientación del fémur la inclinación tibial tomará el valor $\mathbf{q}+\mathbf{a}$.

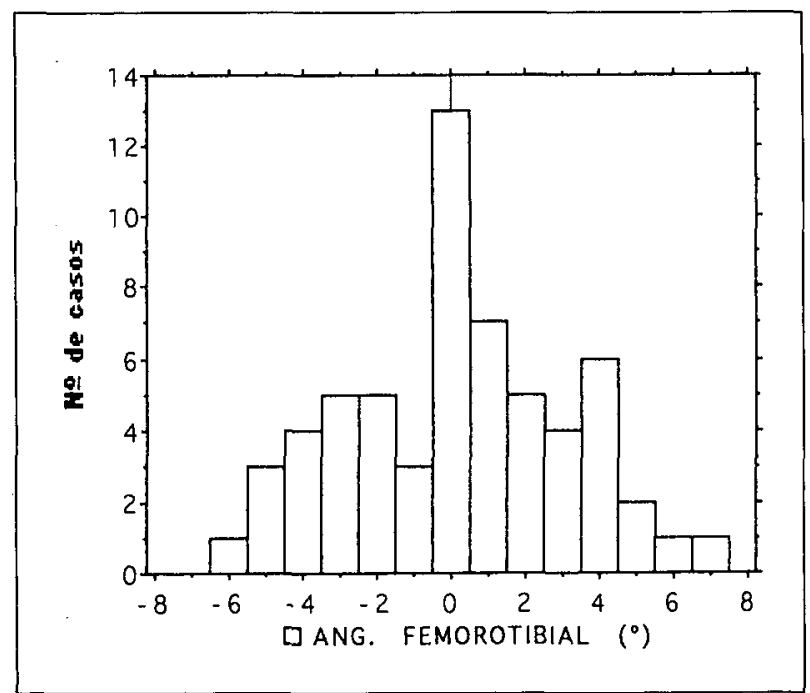

Figura 5. Distribución de frecuencias del ángulo femorotibial. Existió una considerable variabilidad en sus valores, que oscilaron entre un valgo de $-6.25^{\circ}$ y un varo de $7^{\circ}$.

$32.4 \pm 10.4$ años siendo la máxima 49 y la mínima 13. No existieron diferencias significativas entre las edades medias del grupo de mujeres y de varones ni entre ninguna de las variables estudiadas comparando las correspondientes al miembro inferior izquierdo ó derecho. Los valores conjuntos y separados por sexos de los parámetros estudiados, así como el nivel de significación de las diferencias entre los grupos masculino y femenino, se resumen en la tabla 1.

El valor medio del ángulo femorotibial fué de $0.1 \pm$ $3^{\circ}$, con un rango de variación de $13.2^{\circ}$ entre los valores límite; comprendidos entre un mínimo de $-6.2^{\circ}$ y un máximo de $+7^{\circ}$ (fig. 5). Variaciones ligeramente menores se encontraron en los ángulos condíleo y tibial, cuyos valores medios fueron de $87.8^{\circ}$ y $88.4^{\circ}$ respectivamente (fig. 6 y 7). El ángulo de convergencia de la interlínea, cuya media fué de $0.6 \pm 1.7^{\circ}$, varió ente $-4^{\circ} \mathrm{y}$ $4^{\circ}$ (fig. 8). Existió asimismo una considerable dispersión de las proporciones entre las longitudes de los hue-

TABLA I

VALORES TOTALES Y AGRUPADOS POR SEXOS DE LOS ÁNGULOS, DIMENSIONES LONGITUDINALES Y PROPORCIONES

\begin{tabular}{|c|c|c|c|c|c|c|c|c|c|c|}
\hline \multirow[b]{2}{*}{ Variables } & \multicolumn{3}{|c|}{ TOTAL } & \multicolumn{3}{|c|}{ MUUERES } & \multicolumn{3}{|c|}{ VARONES } & \multirow[b]{2}{*}{$p$} \\
\hline & media $\pm S D$ & rango & $n$ & media $\pm S D$ & rango & $n$ & media $\pm S D$ & rango & $n$ & \\
\hline Angulo femorotibial $(\alpha)\left({ }^{\circ}\right)$ & $0,1 \pm 3.0$ & $-6.2 / 7.0$ & 60 & $-1.7 \pm 2.6$ & $-6.2 / 4.0$ & 28 & $1.7 \pm 2.4$ & $-4.0 / 7.0$ & 32 & $<0.001$ \\
\hline Angulo condileo $(\phi)\left(^{\circ}\right)$ & $87.8 \pm 2.0$ & $83.0 / 92.0$ & 60 & $87.6 \pm 2.1$ & $83.0 / 92.0$ & 28 & $88.1 \pm 1.8$ & $83.0 / 91.0$ & 32 & N.S. \\
\hline Angulo tibial $(\tau)\left({ }^{\circ}\right)$ & $88.4 \pm 2.1$ & $84.0 / 93.0$ & 60 & $89.8 \pm 1.5$ & $86.5 / 93.0$ & 28 & $87.1 \pm 1.6$ & $84.0 / 90.0$ & 32 & $<0.001$ \\
\hline Angulo de convergencia $(\beta)\left({ }^{\circ}\right)$ & $0.6 \pm 1.7$ & $-4.0 / 4.0$ & 60 & $0.5 \pm 2.1$ & $-4.0 / 4.0$ & 28 & $0.8 \pm 1.2$ & $-1.0 / 4.0$ & 32 & N.S. \\
\hline Diámetro bifemoral (h) (mm) & $180.7 \pm 10.4$ & $156.0 / 197.5$ & 30 & $183.3 \pm 10.9$ & $156.0 / 197.5$ & 28 & $178.3 \pm 9.4$ & $156.0 / 192.5$ & 32 & $<0.05$ \\
\hline Longitud fémur (f) (mm) & $448.4 \pm 29.8$ & $375.0 / 496.0$ & 60 & $433.1 \pm 32.3$ & $375.0 / 483.0$ & 28 & $461.9 \pm 19.6$ & $432.0 / 496.0$ & 32 & $<0.001$ \\
\hline Longitud tibia (t) $(\mathrm{mm})$ & $368.7 \pm 31.1$ & $299.0 / 431.5$ & 60 & $353.3 \pm 34.8$ & $299.0 / 418.0$ & 28 & $382.2 \pm 19.7$ & $347.5 / 431.5$ & 32 & $<0.001$ \\
\hline Relación t/h & $2.5 \pm 0.2$ & $2.1 / 3.0$ & 60 & $2.4 \pm 0.1$ & $2.1 / 2.6$ & 28 & $2.6 \pm 0.2$ & $2.3 / 3.0$ & 32 & $<0.001$ \\
\hline Relación th & $2.0 \pm 0.2$ & $1.2 / 2.4$ & 60 & $1.9 \pm 0.1$ & $1.7 / 2.3$ & 28 & $2.1 \pm 0.1$ & $1.9 / 2.4$ & 32 & $<0.001$ \\
\hline Relación $(f+f) / h$ & $4.5 \pm 0.4$ & $3.7 / 5.4$ & 60 & $4.3 \pm 0.3$ & $3.7 / 4.9$ & 28 & $4.7 \pm 0.3$ & $4.2 / 5.4$ & 32 & $<0.001$ \\
\hline
\end{tabular}




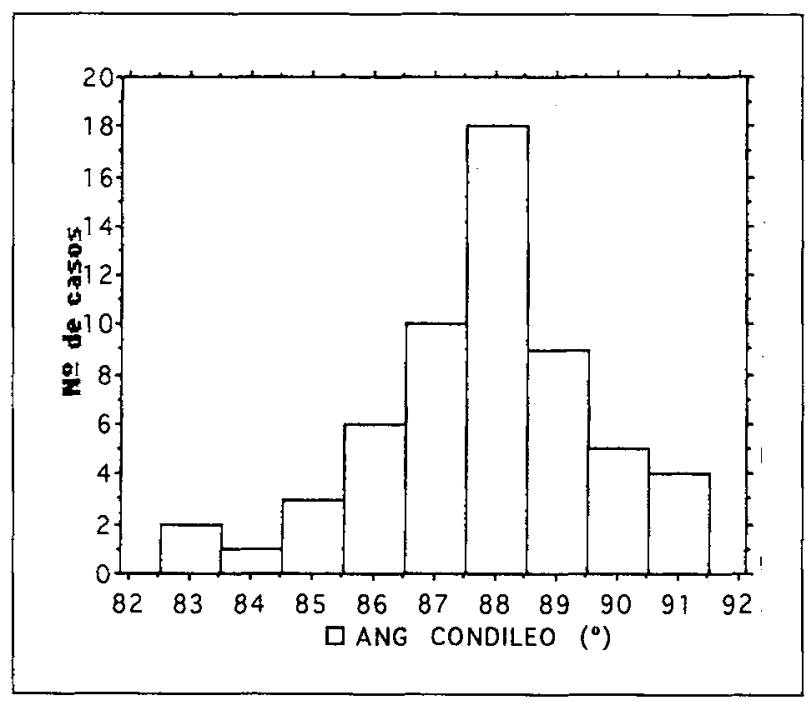

Figura 6. Distribución de frecuencias del ángulo condíleo cuya variación se encontró en el rango entre $83^{\circ}$ y $9^{2}$.

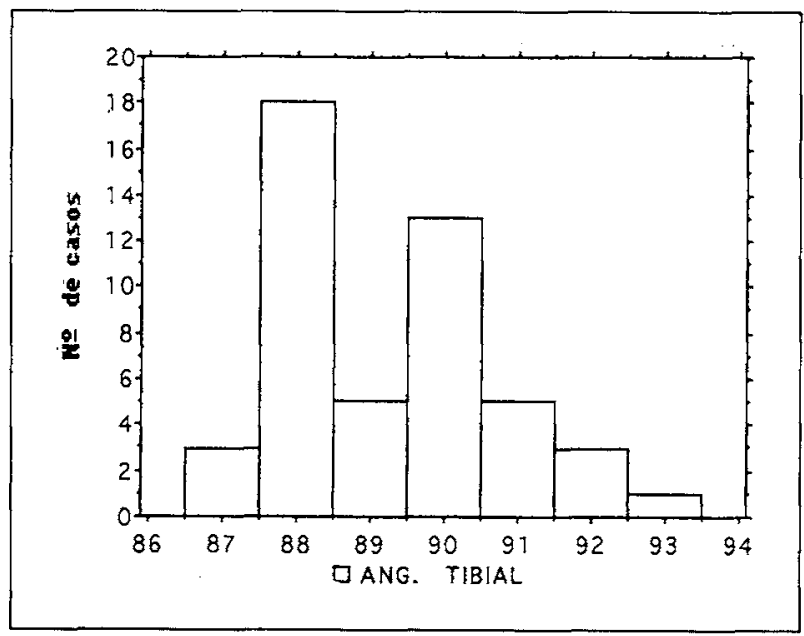

Figura 7. Distribución de frecuencias del ángulo tibial que varió entre $84^{\circ}$ y $93^{\circ}$.

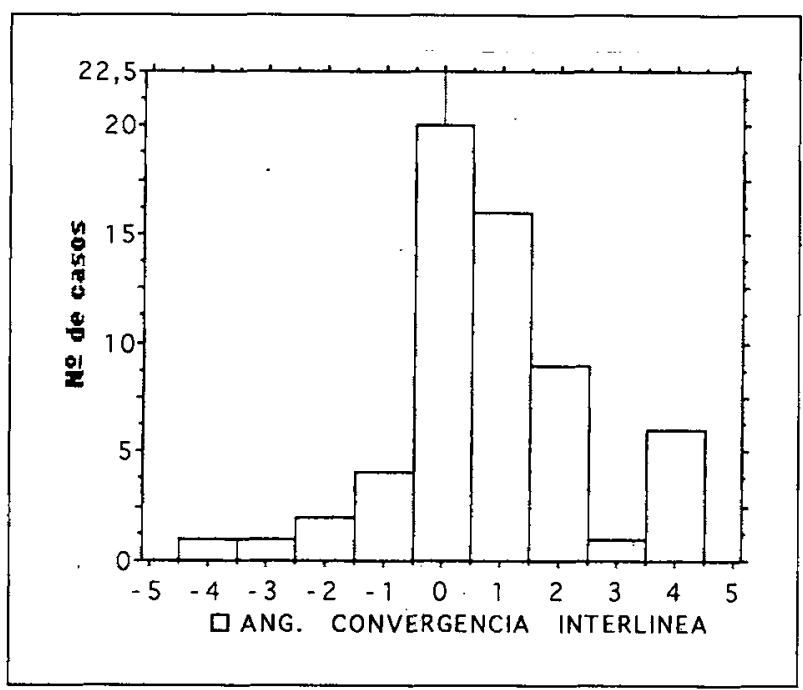

Figura 8. Distribución de frecuencias del ángulo de convergencia articular, que varió desde una apertura medial de $-4^{\circ}$ a una apertura lateral de $4^{\circ}$. sos de la pierna y la anchura de las caderas. Así, la longitud del miembro inferior $(\mathrm{f}+\mathrm{t})$ superó como promedio 4.5 veces la del diámetro bifemoral (h), peró este valor osciló entre un mínimo de 3.7 y un máximo de 5.4 .

Agrupando separadamente a mujeres y hombres pudo apreciarse que existieron diferencias estadisticamente significativas $(\mathrm{p}<0.001)$ entre ambos grupos en las magnitudes medias del ángulo femorotibial y del ángulo tibial $\left(-1.7^{\circ}\right.$ de valgo y casi $90^{\circ}$ de ángulo tibial para las primeras frente a $1.7^{\circ}$ de varo y $87^{\circ}$ de inclinación tibial medial para los segundos). Con las excepciones de los ángulos condíleo (NS) y de convergencia articulara (NS) también se presentaron diferencias en todas las demás variables estudiadas con el mismo nivel de significación $(p<0.001)$ o ligeramente inferior $(p<0.05$, para el diámetro bifemoral).

Los coeficientes de correlación lineal obtenidos del análisis de regresión y sus niveles de significación estadística se sintetizan en la tabla 2. Los ángulos femorotibial y condíleo mantuvieron entre sí una lineal positiva significativa, tanto en los grupos separados de varones y mujeres $(p<0,01)$ como en la muestra conjunta de ambos ( $p<0.001$, fig 9). Se comprobó correlación lineal negativa entre los ángulos femorotibial y tibial en las muestras de varones y conjunta ( $p<0.001$, fig. 10 ), pero no en el grupo femenino. El ángulo femorotibial se correlacionó positivamente con el ángulo de convergencia articular, en la muestra femenina y total $(\mathrm{p}<0.01$, fig 11). El ángulo condíleo y de convergencia presentaron correlación lineal negativa en los varones y en la muestra global $(\mathrm{p}<0,05$ ). En ninguno de los grupos se demostró correlación significativa entre los ángulos condíleo y tibial; ni entre último y la convergencia de la interlínea.

En la muestra de mujeres el ángulo femorotibial se correlacionó negativamente con el diámetro bifemoral (h) y positivamente con las proporciones $(\mathrm{t} / \mathrm{h})$ y $((\mathrm{f}+\mathrm{t}) / \mathrm{h})$ $(\mathrm{p}<0.05)$. No se evidenció correlación significativa entre la magnitud del ángulo femorotibial y las dimensiones del miembro inferior en el grupo aislado del sexo masculino. Sin embargo, en la muestra conjunta se repitieron las correlaciones encontradas en el grupo femenino -negativa con el diámetro bifemoral $(\mathrm{p}<0.05)$; positiva con las proporciones $t / h(p<0.001)$ y $(\mathrm{f}+\mathrm{t}) / \mathrm{h}(\mathrm{p}<$ 0.01 , fig 12) - y además alcanzó significación estadística ( $\mathrm{p}<0,01)$ su correlación positiva con la proporción $\mathrm{f} / \mathrm{h}$. El ángulo condíleo solo mostró estar correlacionado negativamente con el diámetro bifemoral y con la longitud del fémur $(\mathrm{p}<0.05)$ en la mujer. Existió correlación lineal negativa del ángulo tibial con las longitudes del fémur y de la tibia, y con sus relaciones con el diámetro de la pelvis, en el grupo femenino. Estas correlaciones se mantuvieron e incrementaron su nivel de significación estadística cuando al grupo femenino se añadieron los varones para formar una única muestra $(p<0,001)$. $E 1$ ángulo de convergencia articular no se correlacionó con ninguna dimensión lineal de los miembros inferiores.

Existieron diferencias muy significativas (tabla 3) en los ángulos femorotibial y tibial, así como en las dimensiones longitudinales de los miembros inferiores $(\mathrm{p}<$ 0.001 ) y menos significativas en el diámetro bifemoral $(\mathrm{p}<0.05)$ entre las muestras seleccionadas según el 
TABLA 2

VALORES DE LOS COEFICIENTES DE CORRELACIÓN LINEAL ( $r$ ) ENTRE LOS ÁNGULOS DE ALINEACIÓN DE LA RODILLA, LAS DIMENSIONES Y LAS PROPORCIONES DEL ESQUELETO DE LOS MIEMBROS INFERIORES

\begin{tabular}{|c|c|c|c|c|c|c|c|c|c|c|}
\hline & & $A C$ & AT & $A C A$ & D. Bifemoral (h) & Fémur $(f)$ & Tibia $(t)$ & $f / h$ & $t / h$ & $(f+t) / h$ \\
\hline \multicolumn{11}{|c|}{ Angulo Femorotibial } \\
\hline mujeres & $(n=28)$ & $0,545^{\star *}$ & - & $0,494^{* *}$ & $-0,450^{*}$ & 一 & - & - & $0,436^{*}$ & $0,412^{*}$ \\
\hline varones & $(n=32)$ & $0,538^{\star *}$ & $-0,725^{\star * \star}$ & - & - & - & - & - & - & - \\
\hline ambos & $(n=60)$ & $0,515^{\star \star \star}$ & $-0,684^{* * *}$ & $0,357^{* *}$ & $-0,259^{\star}$ & - & $0,312^{*}$ & $0,336^{\star \star}$ & $0,441^{* * *}$ & $0,395^{\star \star}$ \\
\hline \multicolumn{11}{|c|}{ Angulo condíleo (AC) } \\
\hline mujeres & $(n=28)$ & & - & - & $-0,383^{*}$ & $-0,454^{*}$ & - & - & - & - \\
\hline varones & $(n=32)$ & & - & $-0,443^{*}$ & - & - & - & 一 & 一 & - \\
\hline ambos & $(n=60)$ & & - & $-0,272^{*}$ & - & - & - & - & - & 一 \\
\hline \multicolumn{11}{|c|}{ Angulo tibial (AT) } \\
\hline mujeres & $(n=28)$ & & & - & - & $-0,631^{* * *}$ & $-0,610^{* * \star}$ & $-0,539^{\star \star}$ & $-0,491^{* \star}$ & $-0,525^{\star \star}$ \\
\hline varones & $(n=32)$ & & & - & - & - & - & - & - & - \\
\hline ambos & $(n=60)$ & & & - & - & $-0,470^{* * *}$ & $-0,484^{\star \star *}$ & $-0,456^{* * *}$ & $-0,501^{* * *}$ & $-0,489^{* * *}$ \\
\hline \multicolumn{11}{|c|}{ Ang. convergencia (ACA) } \\
\hline mujeres & $(n=28)$ & & & & - & - & - & - & - & - \\
\hline varones & $(n=32)$ & & & & - & - & - & - & - & - \\
\hline ambos & $(n=60)$ & & & & - & - & - & - & - & - \\
\hline
\end{tabular}

Significación estadística: no significativo $(-) ; P<0.05\left(^{*}\right) ; p<0,01\left(^{* *}\right) ; p<0,001\left(^{* * *}\right)$

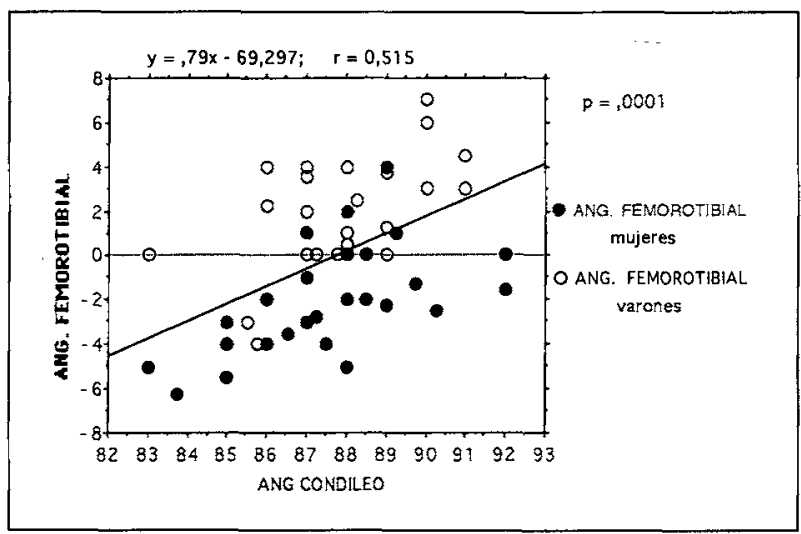

Figura 9. Correlación entre el ángulo femorotibial y el ángulo condíleo. El valgo se suele acompañar de ángulos condíleos inferiores (menor prominencia del cóndilo lateral) que el varo (menor prominencia del cóndilo medial).

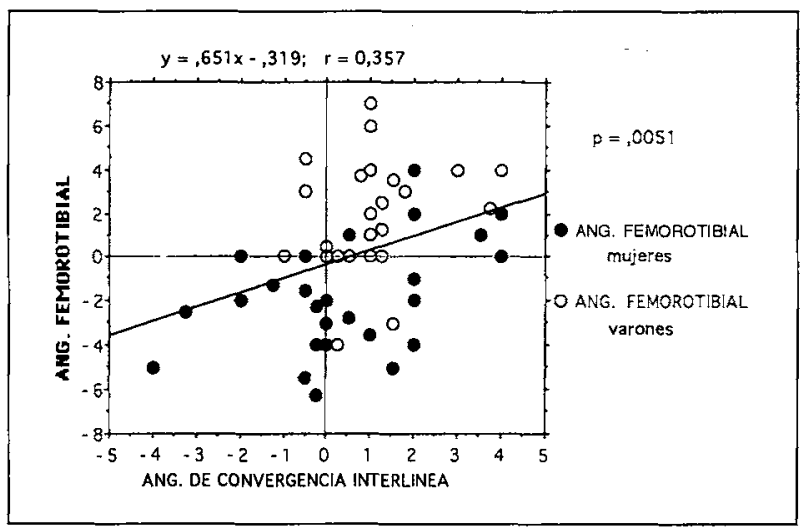

Figura 11. Correlación entre valgo -divergencia medial y varodivergencia lateral de la interlínea.

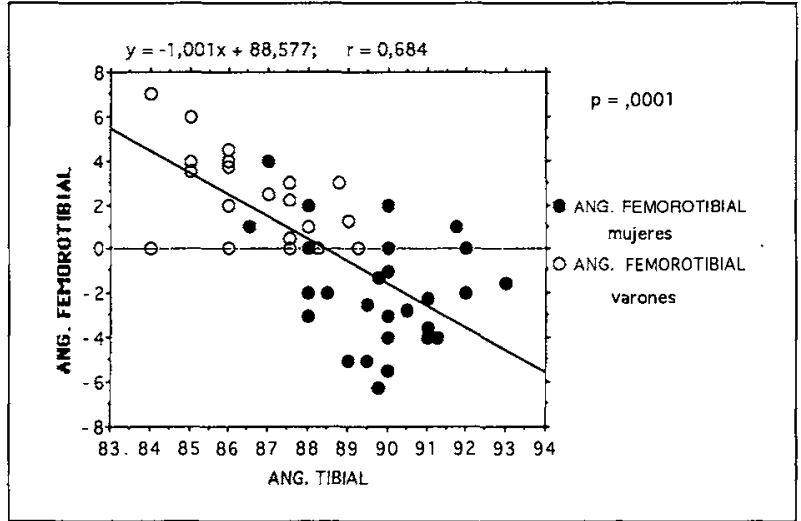

Figura 10. Correlación entre el ángulo femorotibial y el ángulo tibial. Conforme disminuye el varo disminuye la inclinación medial de la línea bituberositaria (aumenta el ángulo tibial).

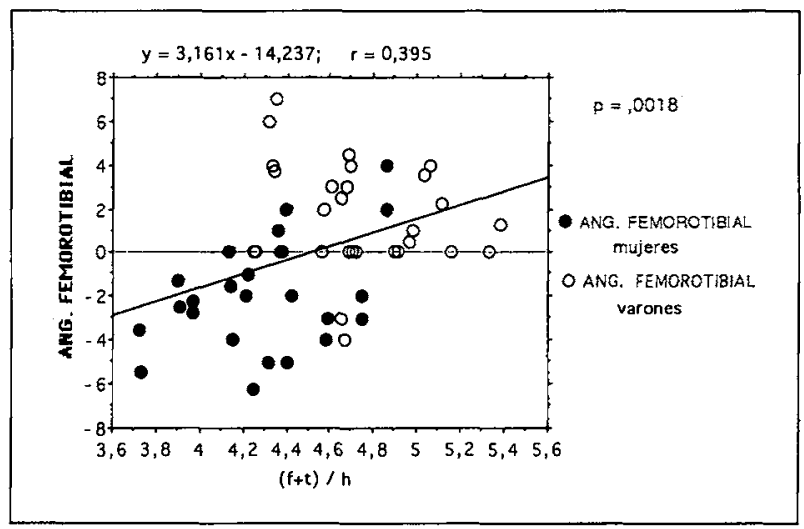

Figura 12. Relaciones entre el ángulo femorotibial y las dimensiones relativas de los miembros inferiores. La alineación envalgo se asocia con las proporciones mas bajas entre la longitud de las piernas $(f+t)$ y la anchura de las caderas $(h)$. Conforme las caderas son proporcionalmente mas estrechas disminuye el valgo y aparece progresivamente el varo. 
TABLA 3

VALORES MEDIOS COMPARADOS DE PARAMETROS DE ALINEACIÓN Y DISTANCIAS

DE LAS REACCIONES DE APOYO ENTRE GRUPOS CUYOS MIEMBROS INFERIORES

PRESENTARON LONGITUDES $(f+t)$ INFERIORES $\left(m_{1}\right)$ O SUPERIORES $\left(m_{2}\right)$ A 4.5 VECES

EL DIAMETRO BIFEMORAL

\begin{tabular}{lccc}
\hline & $\begin{array}{c}m+ \\
(f+t) / h<4.5 \\
n=22\end{array}$ & $\begin{array}{c}m 2 \\
(f+t) / h>4.51 \\
n=22\end{array}$ & \\
\hline Angulo femorotibial $\left({ }^{\circ}\right)$ & media $\pm S D$ & media $\pm S D$ & $p$ \\
Angulo condíleo $\left(^{\circ}\right)$ & $-1,6 \pm 2,8$ & $1,2 \pm 2,2$ & $<0,001$ \\
Angulo tibial $\left({ }^{\circ}\right)$ & $87,8 \pm 2,3$ & $88,3 \pm 1,7$ & $N S$ \\
Angulo convergencia articular $\left({ }^{\circ}\right)$ & $89,6 \pm 1,9$ & $87,6 \pm 1,4$ & $<0,001$ \\
Diámetro bifemoral $(\mathrm{h})(\mathrm{mm})$ & $0,1 \pm 1,9$ & $0,5 \pm 1,2$ & $\mathrm{NS}$ \\
Longitud fémur $(\mathrm{f})(\mathrm{mm})$ & $183,6 \pm 11,9$ & $176,7 \pm 10,3$ & $<0,05$ \\
Longitud tibia $(\mathrm{t})(\mathrm{mm})$ & $424,3 \pm 28,6$ & $472,1 \pm 14,2$ & $<0,001$ \\
Distancia reacción de apoyo $(\mathrm{z})(\mathrm{mm})$ & $340,9 \pm 24,7$ & $389,1 \pm 19,2$ & $<0,001$ \\
Distancia reacción de apoyo $(\mathrm{z})(\mathrm{mm})$ & $10,1 \pm 13,6$ & $15,8 \pm 13,9$ & $\mathrm{NS}$ \\
\hline
\end{tabular}

(*) Distancia calculada igualando a $0^{\circ}$ el ángulo femorotibial

valor $(f+t) / h$ fuese inferior o superior a la media. No se detectó variación apreciable en las distancias de la reacción de apoyo al centro de la rodilla en condiciones naturales $(10,0 \pm 13,6 \mathrm{~mm}$ en $\mathrm{m} 1$ frente a $15,7 \pm 13.9$ $\mathrm{mm}$. en $\left.\mathrm{m}^{2} ; \mathrm{p}=0,0004\right)$. En concordancia con este resultado, no existió correlación entre los valores de $(\mathrm{f}+\mathrm{t}) / \mathrm{h}$ y las distancias de la reacción de apoyo al centro de la rodilla en condiciones naturales (fig. 13); mientras que apareció correlación lineal negativa altamente significativa entre variasbles $(p=0.0001)$ cuando se rectificó a $0^{\circ}$ la alineación entre los ejes mecánicos femoral y tibial (fig 14).

Los valores medios de los ángulos de alineación para distintas constituciones corporales, definidas por proporciones que varían entre los valores 3.0 y 5.8 de la longitud del miembro inferior con la anchura de la pelvis, se expresan en la tabla 4 . Los valores ajustados de los ángulos femorotibial y tibial se despejaron de sus funciones de regresión con la relación $(\mathrm{f}+\mathrm{t}) / \mathrm{h}$; mientras que los correspondientes a los ángulos condíleo y de

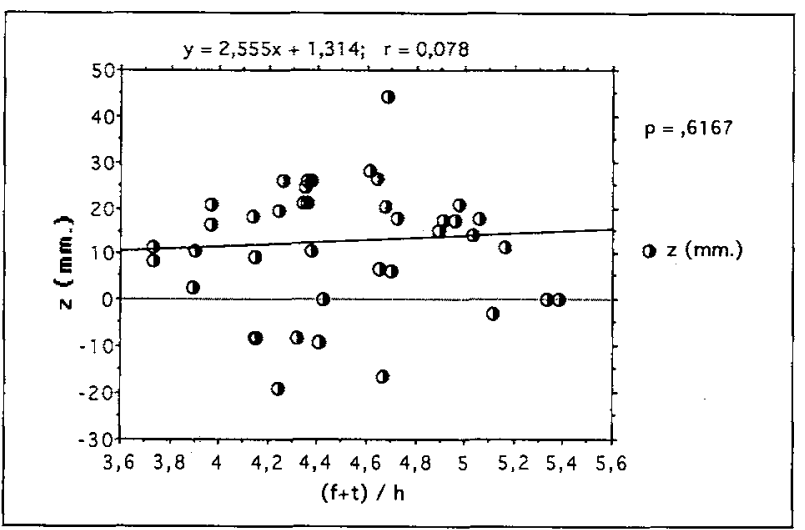

Figura 13. Asusencia de correlación entre als distancias de la reacción de apoyo al centro de la rodilla $(z)$ y las proposiciones entre la longitud del miembro inferior y la anchura de la pelvis en condiciones naturales. convergencia, se dedujeron de sus funciones de regresión con el ángulo femorotibial. Sus valores redondeados, en el caso de paralelismo de las líneas bicondílea y bituberositaria, se presentan en la tabla 5.

\section{DISCUSIÓN}

El valor medio del ángulo femorotibial encontrado en nuestro estudio $\left(0.1^{\circ}\right)$ no difiere significativamente de la alineación a $0^{\circ}$ aceptado en general como criterio de normalidad. La presencia de un rango significativo de variación en los valores de éste ángulo es asimismo coincidente con el señalado por otros autores ${ }^{7,20}$. Sin embargo las diferencias entre mujeres y varones presentes en nuestra serie no se encontraron en estudios pre$\operatorname{vios}^{7}$. Esta discrepancia pudiera obedecer a diferencias antropométricas relacionadas con el distinto origen geográfico y étnico de las poblaciones estudiadas o a variaciones en los métodos de obtención de las telerradiografías ${ }^{2,4}$. Sin embargo, el valor medio de éste parámetro en

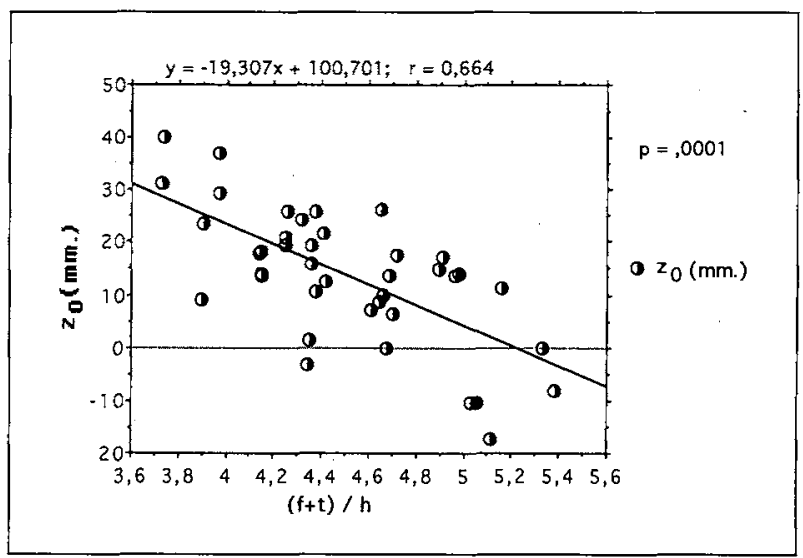

Fifgura 14. Dependencia de la distancia de la reacción de apoyo al centro de la rodilla de los valores de $(f+t) / h$ cuando se sitúan los ejes tibial y femoral en línea recta suprimiendo la variabilidad natural del ángulo femorotibial.. 
TABLA 4

VALORES AJUSTADOS DE LOS ANGULOS DE ALINEACIÓN DE LA RODILLA EN FUNCIÓN DE LA PROPORCIÓN ENTRE LA LONGITUD DEL MIEMBRO INFERIOR $(f+t)$ Y LA ANCHURA DE LA PELVIS $(\mathrm{h})$

\begin{tabular}{ccccc}
\hline & $\begin{array}{c}\text { Angulo } \\
f e m o r o t i b i a l \\
(\alpha)\end{array}$ & $\begin{array}{c}\text { Angulo } \\
\text { condileo } \\
(\phi)\end{array}$ & $\begin{array}{c}\text { Angulo } \\
\text { tibial } \\
(\tau)\end{array}$ & $\begin{array}{c}\text { Angulo } \\
\text { convergencia* } \\
(\beta)\end{array}$ \\
\hline 3 & $-4,753$ & 86,224 & 92,492 & $-0,313$ \\
3,4 & $-3,488$ & 86,649 & 91,424 & $-0,065$ \\
3,8 & $-2,224$ & 87,074 & 90,355 & 0,183 \\
4,2 & $-0,959$ & 87,499 & 89,287 & 0,431 \\
4,6 & 0,305 & 87,925 & 88,219 & 0,679 \\
5 & 1,571 & 88,352 & 87,151 & 0,927 \\
5,4 & 2,834 & 88,775 & 86,083 & 1,175 \\
5,8 & 4,099 & 89,201 & 85,015 & 1,423 \\
\hline
\end{tabular}

$\left.{ }^{*}\right)$ Los valores de los ángulos, obtenidos a partir de las funciones de regresión, no cumplieron exactamente la relación geométrica $\phi-\alpha=\tau-\beta$, manteniendo un error medio de $-0.16 \pm 1.17^{\circ}(n=8)$

TABLA 5

ORIENTACIÓN DE LOS CORTES TIBIAL, FEMORAL Y ÁNGULO FEMOROTIBIAL RESULTANTE SEGÚN EL VALOR DE $(f+t) / h$.

ALINEACIÓN PROTÉSICA. CUADRO GUIA

\begin{tabular}{cccc}
\hline$(f+t) / h^{*}$ & $\begin{array}{c}\text { Corte tibial } \\
(\tau)\end{array}$ & $\begin{array}{c}\text { Corte femoral** } \\
(\phi)\end{array}$ & $\begin{array}{c}\text { Angulo femorotibial } \\
(\alpha)\end{array}$ \\
\hline 3 & 92 & 87 & -5 \\
3,4 & 91 & 87,5 & $-3,5$ \\
3,8 & 90 & 88 & -2 \\
4,2 & 89 & 88 & -1 \\
4,6 & 88 & 88 & 0 \\
5 & 87 & 89 & 1,5 \\
5,4 & 86 & 89 & 3 \\
5,8 & 85 & 4 & 4 \\
\hline
\end{tabular}

$\left({ }^{*}\right)$ Los valores extremos $(3,3.4$ y 5.8$)$ no se presentaron entre los casos estudiados.

$\left(^{\star \star}\right)$ El ángulo del corte femoral fué estimado teniendo en cuenta que para $\beta=0^{\circ}, \phi=\tau+\alpha$ (véase fig. 2).

los varones $\left(1.7^{\circ}\right.$ de varo) y la mayor tendencia al varo en este grupo coinciden con los hallazgos descritos pro Moreland ${ }^{20}$, cuyo estudio se realizó exclusivamente sobre individuos del sexo masculino.

La alineación transversal de la rodilla fue medida con distintos métodos en otros trabajos ${ }^{7,8,20,12,23}$ que en general no tienen en cuenta el efecto de las posibles variaciones de amplitud de la interlínea $\operatorname{articular}^{2,5}$. Sin embargo los valores medios del ángulo condíleo $\left(87.8^{\circ}\right.$ ) y tibial $\left(88.4^{\circ}\right)$ son compatibles con los señalados ${ }^{3}$ en artículos que utilizaron criterios coincidentes. Las diferencias encontradas en este estudio entre los valores del ángulo tibial correspondientes a ambos sexos, son concordantes con las descritas previamente en el ángulo de inclinación de la interlínea7. El valor medio del ángulo tibial en varones $\left(87^{\circ}\right)$-frente a los casi $90^{\circ}$ encontrados en mujeres- se ajusta a la inclinación medial de la interlínea de $3^{\circ}$ publicada anteriormente en varones ${ }^{20}$.

Las diferencias señaladas hasta aquí parecen mostrar que la variabilidad encontada en los valores de los ángu- los femorotibial y tibial en éste y otros estudios guarda relación con el sexo. Así, podría hablarse de un patrón de alineación de «tipo femenino», en el que el ángulo femorotibial suele ser menor de $0^{\circ}$ (valgo ó negativo) y el ángulo tibial mayor de $88^{\circ}$, y de otro «tipo masculino» en el que el ángulo femorotibial tiende a ser superior a $0^{\circ}$ (varo ó positivo) y el ángulo tibial inferior a $88^{\circ}$ (fig. 15).

La presencia de correlación positiva del ángulo femorotibial con el ángulo condíleo (fig. 9) y negativa con el ángulo (fig. 10) expresa, en términos estadísticos, la constatación clínica de que la tendencia al genu valgo suele asociarse a un aplanamiento del cóndilo lateral y femoral y a una inclinación lateral de la superficie articular tibial; mientras que la tendencia al genu varo se acompaña con mayor frecuencia de una aplanamiento del cóndilo femoral medial y a una inclinación medial de la superficie tibial.

La correlaciones encontradas entre el ángulo femorotibial y las dimensiones analizadas, en la serie conjunta 


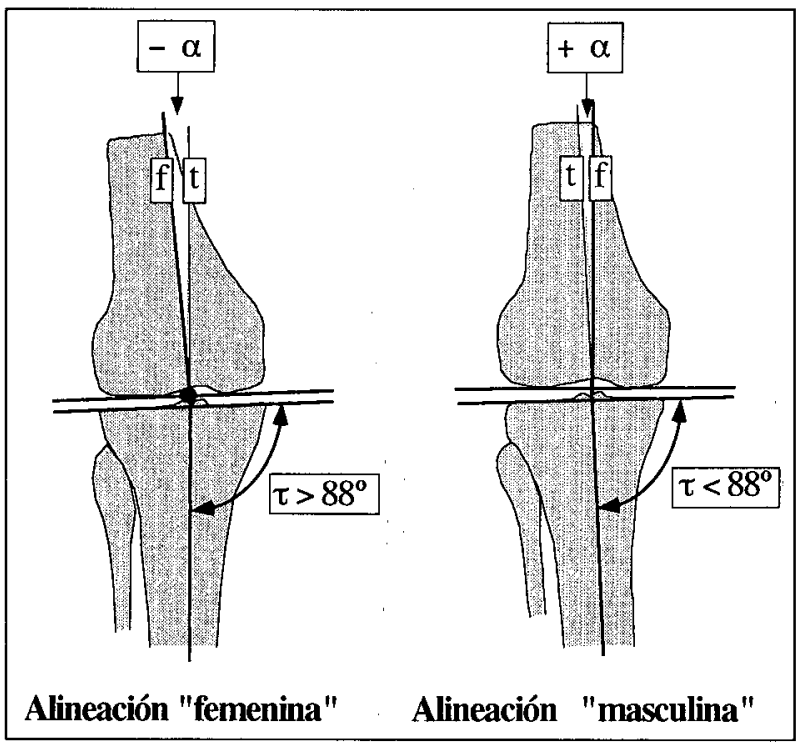

Figura 15. En las mujeres el ángulo femorotibial tendió a ser valgo (a negativo) y varo (a positivo) en los varones; estas diferencias coincidieron con ángulos tibiales superiores e inferiores respectivamente a $88^{\circ}$.

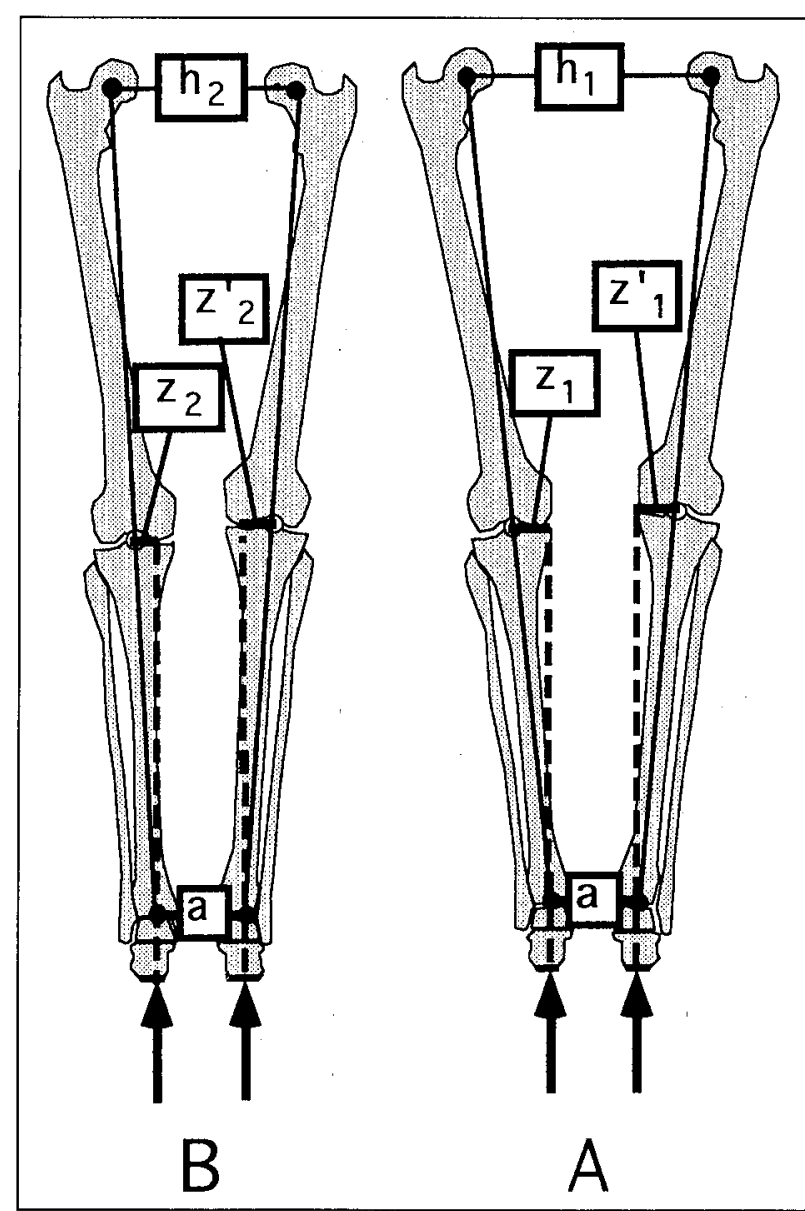

Figura 16. Representación de dos casos con distintas proporciones $(f+t)$ y ángulo femorotibial igual a $0^{\circ}$, en actitud de bipedestación simétrica manteniendo la misma separación entre los tobillos (a). En la mitad derecha de cada figura (A y B) la tibia es mas larga y el fémur mas corto. Dada la verticalidad de las reacciones de apoyo, resulta geometricamente evidente que la magnitud de $z$ depende de la anchura relativa de las caderas $\left(z_{1}>z_{2} ; z_{1}>z_{2}^{\prime}\right)$ y de las longitudes relativas de fémur y tibia $\left(z_{1}>z_{1} ; z_{2}>z_{2}\right)$. sugieren que su valor depende de la proporción entre la longitud de los miembros inferiores y el diámetro bifemoral; sin ambargo, la correlación lineal negativa mostrada en el grupo femenino por el ángulo condíleo tanto con el diámetro bifemoral como con la longitud del fémur parece indicar una relación de éste ángulo con la talla o corpulencia; mientras que el ángulo tibial disminuye conforme aumenta la longitud de los huesos de la pierna o su proporción respecto a la anchura de la pelvis. Estos resultados inducen a pensar que las variaciones en la alineación respectiva de los ejes tibial y femoral y la orientación de las superficies articulares dependen de ciertas dimensiones absolutas y relativas del esqueleto, entre las que parece jugar un papel destacado la proporción entre la longitud del miembro inferior y el diámetro bifemoral $((\mathrm{f}+\mathrm{t}) / \mathrm{h})$. El hecho de que las correlaciones de esta proporción se presentasen en el grupo femenino aislado y requiriesen la unión con el masculino en una sola muestra para alcanzar o incrementar su significación estadística sugieren que la variabilidad de ésta relación puede influir en la alineación con independencia del sexo; en cuyo caso las diferencias observadas serían atribuíbles al predominio de determinadas relaciones antropométricas en las muestras de hombres y mujeres. Así, como muestra la tabla 4, las personas con pelvis mas anchas en relación a la longitud de los miembros inferiores tienden a tener un ángulo femoral en valgo y mayor elevación del platillo tibial interno; dando lugar a una alineación de «tipo femenino». Por el contrario, las personas con pelvis mas estrechas y miembros inferiores mas largos tienden al varo y los platillos tibiales se inclinan en dirección medial, rasgos que definen la rodilla de «tipo masculino».

De los resultados y argumentos presentados en párrafos anteriores parece deducirse que la variabilidad en los ángulos formados por los ejes longitudinales y transversales del miembro inferior no constituye un fenómeno aleatorio, sino que se encuentra vinculada a variaciones de origen sexual, constitucional o epigenético de otros parámetros; por lo que parece plausible afirmar que cada ser humano posee una alineación propia de la rodilla, consecuencia de sus características antropométricas individuales. Sin embargo, bajo el criterio de normalidad generalmente aceptado subyace la engañosa creencia en que el eje mecánico del miembro inferior, trazado desde el centro de la cabeza femoral $(\mathrm{H})$ al centro del tobillo (A), coincide con el «eje de carga» de la rodi$11 a^{5,25}$. Según esta creencia la alineación a $0^{\circ}$ del fémur y la tibia, que por definición obliga al eje mecánico del miembro inferior a pasar por el centro de la rodilla (K? fig. 1), permitiría una distribución simétrica de la carga entre ambos lados de la articulación ${ }^{17,18}$, mientras que la divergencia entre los ejes mecánicos del fémur y de la tibia producirá un desplazamiento correlativo del eje mecánico del miembro inferior $-y$, por lo tanto, del eje de carga- de su posición central, dando lugar a una predominio anormal del soporte de fuerzas por uno $\mathbf{u}$ otro compartimento. Desde esta perspectiva no parecía muy razonable aceptar como natural una variabilidad de la alineación de la rodilla que tendría como consecuencia una sobrecarga mecánica monocompartimental.

Sin embargo, la distancia de la línea de acción de la reacción de apoyo del pie en el suelo -línea a la que se podría denominar «eje e carga» con mayor propiedad 
que al eje mecánico- depende de un parámetro antropométrico individual, la longitud de la pierna y de su inclinación respecto al suelo (fig. 3) la cual constituye una variable postural función del ángulo femorotibial y del grado de abd/adducción de la cadera. Cuando los ejes femoral y tibial se prolongan en línea recta y se permanece en apoyo bipodal simétrico, dicha distancia depende exclusivamente del grado de separación entre los miembros inferiores; por lo que, a nivel interindividual, para una separación constante entre ambos pies su magnitud es función directa de las proporciones geométricas del miembro inferior (fig. 16), lo que parece dar explicación a la elevada correlación encontrada entre ambas variables cunado se simuló la correción del ángulo femorotibial a $0^{\circ}$ (fig. 13). En sentido contrario su ausencia del correlación en condiciones de alineación natural, a pesar de la gran deversidad de configuraciones geométricas presentes en la muestra, sugiere que las variaciones interindividuales en la alineación de la rodilla tienden a mantener las distancias de la reacción de apoyo - y por lo tanto los momentos actuantes- dentro de cierto límites, compensando los efectos geométricos de la variabilidad en las dimensiones longitudinales y transversales del esqueleto de los miembros inferiores; compensación que, en contra de las creencias anteriormente señaladas, resultaría anulada por la rectificación artifical de miembro inferior.

Por estos motivos parecería razonable que, frente a los procedimientos de «alineación clásica» o «alineación anatómica» que pretenden implantar las prótesis en la misma posición en todos los casos, los cortes tibial y femoral se realizasen con la inclinación correspondiente a las características antropométricas individuales, a fin de devolver al miembro inferior su alineación natural. Generalmente, en rodillas susceptibles de sustitución artroplástica de las deformidades secundarias al deterioro articular impiden reconocer los ángulos longitudinales y transversales correspondientes a la alineación previa a la enfermedad; en otras ocasiones la enfermedad articular se establece sobre deformidades previas. En éstas situaciones, conociendo las dimensiones individuales de los miembros inferiores, los valores en la tabla 4 -deducidos de las funciones de regresión de las 60 rodillas estudiadas- podrían servir para estimar la alineación natural mas probable de la rodilla original.

En el caso de las prótesis, mientras sus componentes mantengan contacto bicompartimental, la línea bicondílea y bituberositaria son coincidentes por lo que el ángulo de convergencia articular es igual a cero. Teniendo en cuenta este condicionante, a fin de estimar los valores de la «alineación natural» con fines de planificación preoperatoria, se redondearon los valores angulares medios obtenidos de las funciones de regresión (tabla $5)$. Los valores de los ángulos tibial y condíleo contenidos en dicha tabla, estimados a partir de la relación entre la longitud del miembro inferior y el diámetro bifemoral, facilitan una guía de la orientación de los cortes tibial y femoral que permitiría alcanzar un ángulo femorotibial y una inclinación de la interlínea adaptada a las características antropométricas individuales. La proporción $(\mathrm{f}+\mathrm{t}) / \mathrm{h}$ puede calcularse simplemente tras medir en una telerradiografía de los miembros inferiores la distancia entre los centros de ambas caderas y las longitudes de los ejes mecánicos del fémur y la tibia.

La ampliación del estudio a una población mas numerosa y la profundización en el conocimiento de las cau- sas y consecuencias de la variabilidad en la alineación de la rodilla, objeto de este trabajo, podría ser útil para mejorar nuestra compresión de la fisiopatología de la rodilla y de sus técnicas de reparación.

\section{AGRADECIMIENTOS}

Deseo destacar la generosidad del personal del Hospital do Meixoeiro que se prestó voluntario para la realización de éste estudio; agradezco en particular la cooperación de los técnicos radiológicos y de los doctores Roberto Casal, Jorge González y Luis Montero.

\section{BIBLIOGRAFÍA}

1. Bargen, J.H.; Blaha, J.D.; Freeman, M.A.: «Alignment in Total Knee Arthropolasty. Correlated Biomechanica! and Clinical Observations», Clin. Orthop:; 173; 178-83; 1983

2. Casal, R; Montero, L.; González, J.: Pichel, C.M: «Alineación frontal de la rodilla. Concepto y determinación de la posición reutra en el vivo», Biomecáncia éste número.

3. Cooke, D.V.; Pinchora, D.; Siu, D.; Scudamore, R.A.; Bryant, J.T.: «Surgical Implications of Varus Deformity of the Knee with Obliquity of Joint Surfaces», J. Bone Joint Surg.; 71-B; 560-565; 1989.

4. Dehnan, R.A.: «Radiological Examination of the Knee Joint and Other Special Investigations», en Freeman, M A.R.: «Arthritisof the Knee», pp. 77-109; Springer - Verlag Berlin Heidelberg New York, 1980.

5. Dugdale, T.W.; Noyes, F.R.; Styer, D.: «Preoperative Planning for High Tibial Osteotomy. The Effect of Lateral Tibiofemoral Separation and Tibiofemoral Lenght», Clin. Orthop; 274; 248-64; 1992.

6. Goldberg, V.M.; Figgie, H.E.; Figgie, M.P.: «Technical Considerations in Total Knee Surgery. Management of patella problems»; Orthop. Clin. North. Am.; 20,2; 189-99;1989.

7. Hsu, R.W.W.; Himeno, S.; Conventry, M.B.; Chao, E.Y.S.: «Normal Axial Alignment of the Lower Extremity and Load-Bearing Distribution at the Knee», Clin. Orthop.; 255; 215-227; 1990.

8. Hungerford, D.S.; Krakow, K.A.; Kenna, R.V.: «Alignment in Total Knee Arthroplasty». En Dorr; «The Knee: Papers of the First Scientific Meeting of the Knee Society", pp. 9-21; University Park Press; Baltimore, 1985.

9. Insall, J.N.; Binazzi, R.; Soudry, M.; Mestriner, L.A: «otal Knee Arthroplasty», Clin Orthop; 192; 13-22; 1985.

10. Insall, J.N.; Hood, R.W.; Flawn, L.D.; Sullivan, D.J.: «The Total Condylar Knee Prosthesis in Gonarthrosis»; J. Bone Joint. Surg.; 65-A; 619-27; 1983.

11. Jonsson, B.: Astrom, J.: «Alignment and long-term Clinical Results of a Semiconstrained Knee Prsothesis». Clin. Orthop.; 226; 124-28; 1988.

12. Kapandji, I.A.: «Los ejes de la articulación de la rodilla» en «Cuadernos de fisiología articular», pag. 74; Toray Massoon, Barcelona, 1970.

13. Kennedy, W.R.; White, R.P.: "Unicompartimental Arthroplasty of the Knee. Postoperativa alignment and its Influence en Overal Results», Clin. Orthop. $221 ; 278-85 ; 1987$.

14. Kettelkamp, D.B.; Chao, E.Y.S.: «A Method for Quantitativa Analysis of Medial and Lateral Compression Forces at the Knee During Standing», Clin. Othop.; 83; 202-213; 1972.

15. Krakow, K.A.: «The Technique of Total Knee Arthroplasty», Chap. 5; C.V.Mosby Company, 1990.

16. Laskin, R.S.: «Varus Knee Deformities: A Review of Ten Years Experience», en "Total Arthroplasty of the Knee: Proceedings of the Knee Society, 1985-1986», Aspen Publishers; Rockeville, Maryland, 1987.

17. Maquet, P.; Simonet, J.; de Marchin, P.: «Biomécanique du genou et gonartrose», rev. Chir. Orthop; 53,2; 11-38; 1967.

18. Maquet, P.G.J.: «Biomechanics of the Knee», Springer-Verlag; Berlin Heidelberg New York, 1976.

19. Mathews, L.S.; Goldstein, S.A.; Kaufer, H.: «Experience with three Distinct Types of Total Knee Joint Arthroplasty), Clin. Orthop: 192; 97-107; 1985.

20. Moreland, J.R.: «Mechanisms of failure in Total Knee Arthroplasty», Clin. Orthop.; 226; 49-64; 1988.

21. Moreland, J.R.; Basset, L.W; Hanker, G.J.: «Radiographic Analysis of the Axial Alignment of the Lower Extremity), J. Bone. Joint Surg.; 69-A; 745-749; 1987

22. Rand, I.A.; Conventry, M.B.; «Ten-year Evaluation of Geometric Total Knee Arthroplasty»; Clin. Orthop.: 232; 168-73; 1988 .

23. Steindler, A.: «Kinesiology of the Human body»; p. 330.; Charles C. Thomas; Springfield, 1955.

24. Tew, M; Waugh, W.: «Tibiofemoral Alignment and the Results of Knee Replacement», J. Bone Joint. Surg.; 67-B; 551-66; 1985.

25. Volz, R.G.; Kantor, S.G.; Howe, C.; Mc Murtry, M.: «Factors Affecting Tibial Component Stability: A Comparative Study» En «Total Arthroplasty of the Knee: Proceedings of the Knee Society, 1985-1986»; pp. 109-117.; Aspen Publishers, Rockville, Maryland; 1987. 